\begin{tabular}{c} 
International Journal of Engineering \& Technology, $7(4)(2018) 6657-6664$ \\
International Journal of Engineering \& Technology \\
SPC \\
Website: $\begin{array}{c}\text { www.sciencepubco.com/index.php/IJET } \\
\text { doi: } 10.14419 / \text { ijet. } v 74.2464 \\
\text { Research paper }\end{array}$ \\
\hline
\end{tabular}

\title{
Improvement of a nonnegative preserved efficient solver for atmospheric chemical kinetic equations
}

\author{
Atika RADID $^{1 *}$ and Karim RHOFIR ${ }^{2}$ \\ ${ }^{1}$ University Hassan II Casablanca FSAC-MACS, BP. 5366, Maarif Casablanca, MOROCCO \\ ${ }^{2}$ University Sultan Moulay Slimane ENSA-LISERT Bd. Beni Amir, BP. 77, Khouribga, MOROCCO \\ *Corresponding authorE-mail: atikaradid@gmail.com
}

\begin{abstract}
Generally, chemical reactions from atmospheric chemistry models are described by a strongly coupled, stiff and nonlinear system of ordinary differential equations, which requires a good numerical solver. Several articles published about the solvers of chemical equations, during the numerical simulation, indicate that one renders the concentration null when it becomes negative. In order to preserve the positivity of the exact solutions, recent works have proposed a new solver called Modified-Backward-Euler (MBE). To improve this solver, we propose in this paper an iterative numerical scheme witch is better fitted to stiff problems. This new approach, called Iterative-Modified-Backward-Euler (IMBE), is based on iterative solution of the P-L structure of the implicit nonlinear ordinary differential equations on each time step. The efficiency of the iteration process is increased by using the Gauss and Successive-Over-Relaxation (SOR). In the case of fast/slow chemical kinetic reactions, we proposed an other variant called Iterative-Quasi-Steady-State-Approximation (IQSSA). The numerical exploration of stiff test problem shows clearly that this formalism is applicable to a wide range of chemical kinetics problems and give a good approximation compared to the recent solver. The numerical procedures give reasonable accurate solutions when compared to exact solution.
\end{abstract}

Keywords: Atmospheric Chemical kinetic, Stiff ODE's, Positivity preservation, Iterative-Modified-Backward-Euler, Iterated QSSA.

\section{Introduction}

The tools used in human activities are a major force affecting the chemical composition of the Earth's atmosphere. We are now witnessing a climate change that is causing significant damage. Air pollution forecast is becoming more and more important nowadays to predict some climate change. Chemical kinetic equations is an important component of air pollution models. Generally, chemical reactions from atmospheric chemistry models are described by a strongly coupled, stiff and nonlinear system of ordinary differential equations, which requires a good numerical solver. The difficulty in treating these stiff problems is the stability limitation placed on the step-size of explicit schemes [12]. This limitation usually forces the use of an implicit scheme which requires the solution of a nonlinear system of equations at each step.

Over the past decades, numerous papers about chemical equation solvers have been proposed such as Quasi-Steady-State-Approximation (QSSA), Modified QSSA, HYBRID, Modified HYBRID, LSODE, GEAR, ...etc (cf [1], [2],[3], [5] and there related references). However, these solvers cannot preserve the nonnegativity of the exact solutions. Therefore, in the calculation, the negative numerical concentration values are usually set to zero artificially, which may cause simulation errors.

Recently, Fan Feng et all [2], [3] proposed a new method that can unconditionally preserve the nonnegativity of the exact solutions called Modified-Backward-Euler (MBE) algorithm which is much faster and more precise than the traditional solvers such as LSODE and QSSA. In this paper, we propose an improvement approach of this algorithm called Iterative-Modified-Backward-Euler (IMEB). Then, we examine a methodology for combining implicit solution techniques with iterative methods in a novel way to produce new iterative time differencing methods with enhanced stability characteristics. This approach use one step integration method and composed on two steps numerical schemes. The first one, is to give an improvement of MBE by using an iterative approach for full implicit system which is better fitted than MBE for stiff problem, and the second hand to define a Gauss-Seidel and Successive over relaxation fixed point iterations associated to these to approach.

The paper is organized as follows: we first present in section 2 a mathematical modelling as preliminaries on nonlinear stiff system of differential equations arising from chemical atmospheric problem. In section 3 we first formulate Iterative-Modified-Backward-Euler method and then introduce the Gauss-Seidel and Successive Over Relaxation variants and the technical adapted step-size. To take into account the case of Fast-Slow reaction, we introduce in order section 4 the Iterative-Quasi-Steady-State-Approximation method. Numerical illustrations are provided in section 5, and conclusions are given in section 6. 


\section{Mathematical modelling}

In order to give the user a simple writing of the mathematical formulation easy to implement in a digital setting; we give in this section the different models of chemical kinetics problems.

Most Chemical Reaction (CR) systems are usually represented by a set of elementary chemical reactions, rated $r_{i}$ for $i=1,2, \ldots ;$ for example :

$$
\begin{aligned}
& r_{1}: B_{1} \longrightarrow A_{1} \\
& r_{2}: A_{1}+A_{2} \longrightarrow 3 A_{1} \\
& r_{3}: B_{2}+A_{1} \longrightarrow A_{2}+B_{3} \\
& r_{4}: A_{1} \longrightarrow B_{4}
\end{aligned}
$$

and each elementary chemical reaction is given by :

$$
\sum_{k=1}^{n} \alpha_{k}^{s} A_{k}+\sum_{k=1}^{l} \beta_{k}^{s} B_{k} \stackrel{k_{q, s}}{\longrightarrow} \sum_{k=1}^{n} \alpha_{k}^{q} A_{k}+\sum_{k=1}^{l} \beta_{k}^{q} B_{k}
$$

where $A_{1}, A_{2}, \ldots$ and $B_{1}, B_{2}, \ldots$ designate the molecular chemical species of each RC system. The $B_{j}$ denoting external species whose concentration is constant or known. An RC system with species external is said to be open, otherwise it is said to be closed.

The constants $k_{q, s}$ designate rate of each reaction constants and the coefficients $\alpha_{k}^{s}, \beta_{k}^{s}$ are integers and can be null.

If the external system species are removed from the reactions, then any elementary chemical reaction will be written as :

$$
\alpha_{1}^{s} A_{1}+\alpha_{2}^{s} A_{2}+\ldots+\alpha_{n}^{s} A_{n} \longrightarrow \alpha_{1}^{q} A_{1}+\alpha_{2}^{q} A_{2}+\ldots+\alpha_{n}^{q} A_{n}
$$

\subsection{Stoichiometric space}

Definition 1. Complex will be called any linear combination of chemical species that to the right or left of the symbol $(\longrightarrow)$.

The complexes of the previous example are :

$B_{1}, A_{1}, B_{2}+A_{1}, A_{1}+A_{2}, 3 A_{1}, A_{2}+B_{3}, B_{4}$

Let $\mathrm{NC}$ be the set of indices of the complexes and NE the set of indices of the chemical species defined as:

$N E=\{1, \ldots, n\} \quad N C=\left\{1, \ldots, n_{c}\right\}$

where $n, n_{c}$ represent respectively the numbers of species and complexes of CR systems. Generally, these two integers are different due to the presence of networking species (i.e species engaged in more than one elementary reaction).

Any elementary reaction (3) will be represented by :

$C(i) \longrightarrow C(j)$

where $i$ and $j$ two indices in NC and $C(i)$ and $C(j)$ symbolize two distinct complexes.

Generally, the number of chemical species is different from the number of complex. Each complex $C(s)$ of the system was :

$C(s)=\alpha_{1}^{s} A_{1}+\alpha_{2}^{s} A_{2}+\ldots+\alpha_{n}^{s} A_{n}$

which we associate the following vector :

$\alpha^{s}=\left(\alpha_{1}^{s}, \alpha_{2}^{s}, \ldots, \alpha_{n}^{s}\right)^{t}$

Let $S$ a subspace of $\mathbf{R}^{n}$ defined by :

$S=E \operatorname{sp} \alpha^{i}-\alpha^{s}$ such that $C(i) \longrightarrow C(s)$ exists

$S$ is called the Stoichiometric space.

\subsection{Complex matrix and kinetic matrix}

Let $\alpha^{1}, \ldots, \alpha^{n_{c}}, n_{c}$ vectors associated with the complex systems. We denote by $Y$ the matrix $\left(n_{c} \times n\right)$ which the $i^{\text {th }}$ column is the vector $\alpha^{i}$. $A$ is called the complex matrix of the CR system.

Let $K$ the matrix $\left(n_{c} \times n_{c}\right)$ whose coefficients $k(i, j)$ are given by :

$$
\begin{aligned}
k(i, j) & =\text { constant rate of the reaction } C(j) \longrightarrow C(i) \text { if it exists } \\
& =0 \quad \text { if not }
\end{aligned}
$$

$K$ is called the kinetic matrix of the CR system. 


\subsection{Vector species training}

Let $u(t)=\left\{u_{1}(t), \ldots, u_{n}(t)\right\}^{t} \in C\left([0, T], \mathbf{R}^{n}\right)$ where $u_{i}(t)$ denotes the concentration of the specie $A_{i}$ at time $t$.

All previous CR system leads to the system of differential equations defined as follow :

$\left\{\begin{array}{l}\frac{d u}{d t}=F(u, t)=\sum_{q, s} r_{q, s}(u) v_{q, s} \\ u(0)=u^{0} \text { given. }\end{array}\right.$

where

$v_{q, s}=\alpha(q)-\alpha(s)$ and $\alpha(e)=\left(\alpha_{1}^{e}, \ldots, \alpha_{n}^{e}\right)^{t}$

and

$r_{q, s}(X)=k_{q, s}\left(\prod_{k=1}^{n} u_{k}^{\alpha_{k}^{s}}\right)\left(\prod_{k=1}^{l}\left[B_{k}\right]^{\beta_{k}^{s}}\right)$

In order to write the equation (10) in the P-L structure form, we define the training vector application $F$ as follow :

$\left\{\begin{array}{l}\frac{d u}{d t}=F(u, t)=P(u, t)-L(u, t) u \\ u(0) \text { given. }\end{array}\right.$

which is also written as :

$\left\{\begin{array}{l}\frac{d u_{i}}{d t}=P_{i}(u, t)-L_{i}(u, t) u_{i} \text { for } i \in\{1, \ldots, n\} \\ u_{i}(0) \text { given. }\end{array}\right.$

where, the vector $P$ and the diagonal matrix $L$ are defined by :

- The term $P_{i}(u, t)=\sum_{q, s} r_{q, s}(u) \alpha_{i}^{q}$ is the production term of the reaction.

- The term $L_{i}(u, t) u_{i}=\sum_{q, s} r_{q, s}(u) \alpha_{i}^{s}$ is the loss term of the reaction.

For the matrix writing, we define the training vector application $F$ as follow :

$$
\begin{aligned}
F: & \mathbf{R}^{n} \rightarrow \mathbf{R}^{n} \\
& u \rightarrow F(u)=A .\left(K-d g\left(K^{t} \cdot 1\right)\right) \cdot u^{A}
\end{aligned}
$$

where $u^{A}=\left(u^{\alpha^{i}}, \ldots, u^{\alpha^{n_{c}}}\right)^{t} ; u^{\alpha^{s}}=u_{1}^{\alpha_{1}^{s}} u_{2}^{\alpha_{2}^{s}} \ldots u_{n}^{\alpha_{n}^{s}}$ and $1=(1, \ldots, 1)^{t}$ and $d g\left(K^{t} .1\right)$ denotes the diagonal matrix. The species of the training vector is given by (15).

1. $Y . d g\left(K^{t} .1\right) \cdot u^{Y}$ is the loss term.

2. Y.K. $u^{Y}$ is the production term.

To find the training vector species (10), we set

$F(u)=\sum_{i, j} k(i, j) u^{\alpha^{j}}\left(\alpha^{i}-\alpha^{j}\right)$

where $k(i, j)$ denote the coefficients of the kinetic matrix.

\section{Iterative-Modified-Backward-Euler method (IMBE) and its variants}

Let consider a subdivision $\left(t_{i}\right)_{i=0}^{i=m}$ strictly increasing such that $t_{0}=0$ and $t_{m}=T$ ([0,T] is the time interval) with step-size $\Delta t$ such that $t_{k+1}=t_{k}+\Delta t$ and $I_{k}=\left[t_{k}, t_{k+1}\right]$.

By the $P$ and $L$ definition in (14) or in (15), we have : for $i=1,2, \ldots, n P_{i} \geq 0, L_{i} \geq 0$. And in each time step interval $I_{k}$ for $k=0, \ldots, m-1$, we have to resolve the following implicit differential equation :

$\left\{\begin{array}{l}\frac{d u}{d t}=F(u, t)=P(u, t)-L(u, t) u \\ u\left(t_{k}\right) \text { given }\end{array}\right.$ 


\subsection{Iterative-Modified-Backward-Euler method (IMBE)}

Using the following notations : Assume known $u_{i}^{k}$ an approximation of $u_{i}\left(t_{k}\right)$, we get $u_{i}^{j+1}$ the approximation of $u_{i}\left(t_{k+1}\right)$ in $I_{k}$ by :

$\frac{u_{i}^{k+1}-u_{i}^{k}}{\Delta t}=F_{i}^{k+1}=P_{i}^{k+1}-L_{i}^{k+1} u_{i}^{k+1}$

where

$P_{i}^{k+1}=P_{i}\left(u_{1}^{k+1}, \ldots, u_{n}^{k+1}\right), L_{i}^{k+1}=L_{i}\left(u_{1}^{k+1}, \ldots, u_{n}^{k+1}\right)$ and $F_{i}^{k+1}=F_{i}\left(u_{1}^{k+1}, \ldots, u_{n}^{k+1}\right)$

In order to define the Modified-Backward-Euler method [2] and [3], we suppose that, for $t \in\left[t_{k}, t_{k+1}\right]=I_{k}$ :

$P_{i}(u(t))-L_{i}(u(t)) u_{i}(t) \sim P_{i}\left(u^{k}\right)-L_{i}\left(u^{k}\right) u_{i}(t)$

Then we have :

$$
\begin{aligned}
& \frac{u_{i}^{k+1}-u_{i}^{k}}{\Delta t}=P_{i}^{k}-L_{i}^{k} u_{i}^{k+1} \\
& u_{i}^{k+1}=\frac{u_{i}^{k}+\Delta t P_{i}^{k}}{1+\Delta t L_{i}^{k}}
\end{aligned}
$$

This scheme presents two major advantages :

- the uncoupled evaluation of the components $u_{i}\left(t_{k+1}\right)$,

- the conservation of positivity.

The implicit euler method associated to (18) is defined by the iterative fixed point iteration as follow : In each time step interval $I_{k}$, let consider the fixed point application $T^{1}$ in $C\left(I_{k+1}, \mathbb{R}^{n}\right)$ defined by :

$T^{1}(v)=w$ where $w$ is a solution of the follow problem :

$\frac{w-u^{k}}{\Delta t}=F(v)$ where $v$ in neighbor of $u^{k}$.

Suppose that the function $F$ is $\mu$-Lipschitz then we have the following proposition :

Proposition 1. If $\Delta t$ is chosen such that $\Delta t \mu<1$, then the fixed point application $T^{1}$ is an contraction with constant of contraction less than 1.

Proof 1. By definition of the fixed point application $T^{1}$, for $w$ and $\widetilde{w}$ such that $w=T^{1}(v)$ and $\widetilde{w}=T^{1}(\widetilde{v})$ then with a simple calculation, we show that $\|w-\widetilde{w}\| \leq \Delta t \mu\|v-\widetilde{v}\|$ and if $\Delta t$ is such that $\Delta t \mu<1$ then $T^{1}$ is contraction with respect to the uniform norm $\|$.$\| .$

To respect the notation of the successive iterative methods, the fixed point application $T^{1}$ can be defined by :

$\left\{\begin{array}{l}v^{l+1}=T^{1}\left(v^{l}\right) \\ v^{0}=u^{k} \text { for all } l=0,1, \ldots\end{array}\right.$

Finally, the fixed point application $T^{1}$ have an fixed point noted $u^{k+1}$, solution of (17) at $t_{k+1}$ and gradually, we calculate all the approximations of the vector u over the interval $[0, T]$.

But this choice of fixed point application has major drawbacks. In some cases, $\Delta t$ is very small and in practice the constant $\mu$ is too large and therefore $\Delta t$ will be close to 0 . On the other hand, this application does not retain positivity.

For these reasons, we propose here a new method that allows the conservation of positivity :

The Iterative-Modified-Backward-Euler is an iterative fixed point iteration defined by

$\left\{\begin{array}{l}w=T(v) \\ v \text { neighbour of } u^{k}\end{array}\right.$

where $w$ is solution of the following equation :

$$
\begin{aligned}
& \text { For } i=1, \ldots, n \\
& \left\{\begin{array}{l}
\frac{w_{i}-u_{i}^{k}}{\Delta t}=P_{i}(v)-L_{i}(v) w_{i} \\
v \text { neighbour of } u^{k}
\end{array}\right.
\end{aligned}
$$

The fixed point of application is therefore defined as follows :

$$
\left\{\begin{array}{l}
v^{l+1}=T^{1}\left(v^{l}\right) \\
v^{0}=u^{k} \text { for all } l=0,1, \ldots
\end{array}\right.
$$

where $v^{*}$, the fixed point of $T$, is solution of the problem (24)

We have :

$\frac{w_{i}-u_{i}^{k}}{\Delta t}=P_{i}(v)-w_{i} L_{i}(v)$

which can be written as :

$w_{k}=\frac{u_{k}^{j}+\Delta t P_{k}(v)}{1+\Delta t L_{k}(v)}$

By the definition of $u, P$ and $L$, the $u_{i}^{k}, P_{k}$ and $L_{k}$ are non-negative. Then we have the following properties : 
i) The fixed point iteration $T$ preserve the positivity.

ii ) The fixed point of application $T$ is contracting if the condition of the proposition 1 is verified.

iii ) The Modified-Backward-Euler method corresponds to a single iteration of the fixed point application $T$.

\subsection{Some variants of the IMBE method}

The system of differential equations (17) are a large coupled model and can be decoupled by applying the iterative fixed point as Jacobi, Gauss-Seidel and Successive-Over-Relaxation (SOR). In the literature [17], [12], many authors use these scheme to reduce computational cost, to accelerate the convergence or to give a good approximation of the solution.

The previous fixed point iteration IMBE correspond to the modified Jacobi method applied to (17).

The Gauss-Seidel IMBE is an iterative fixed point iteration defined by

$$
\left\{\begin{array}{l}
w=T^{G S}(v) \\
v \text { neighbour of } u^{k}
\end{array}\right.
$$

where $w$ is solution of the following equation :

$$
\left\{\begin{array}{l}
\frac{w_{1}-u_{1}^{k}}{\Delta t}=P_{1}\left(v_{1}, \ldots, v_{n}\right)-L_{i}\left(v_{1}, \ldots, v_{n}\right) w_{i} \\
\text { and for } i=2, \ldots, n \\
\frac{w_{i}-u_{i}^{k}}{\Delta t}=P_{i}\left(w_{1}, . ., w_{i-1}, v_{i}, . ., v_{n}\right)-L_{i}\left(w_{1}, . ., w_{i-1}, v_{i}, . ., v_{n}\right) w_{i} \\
v \text { neighbour of } u^{k} .
\end{array}\right.
$$

and the SOR IMBE is an iterative fixed point iteration defined by

$$
\left\{\begin{array}{l}
w=T^{S O R}(v) \\
v \text { neighbour of } u^{k}
\end{array}\right.
$$

where $w$ is solution of the following equation :

$$
\left\{\begin{array}{l}
\frac{w_{1}-u_{1}^{k}}{\Delta t}=P_{1}\left(v_{1}, \ldots, v_{n}\right)-L_{i}\left(v_{1}, \ldots, v_{n}\right) w_{1} \\
\text { and for } i=2, \ldots, n \\
\frac{\bar{w}_{i}-u_{i}^{k}}{\Delta t}=P_{i}\left(w_{1}, . ., w_{i-1}, v_{i}, . ., v_{n}\right)-L_{i}\left(w_{1}, . ., w_{i-1}, v_{i}, . ., v_{n}\right) w_{i} \\
w_{i}=\omega v_{i}+(1-\omega) \bar{w}_{i} \\
v \text { neighbour of } u^{k} .
\end{array}\right.
$$

where $\omega$ is the relaxation parameter.

\subsection{Step-size adaptation}

In order to improve the solver proposed in [2]-[3], we will adopt the same control of the step-size which is defined in the following way : if $\left(\left|P_{k}-L_{k} u_{k}\right|>\right.$ critical rate) then

$\Delta t_{k+1}=\frac{\Delta t_{k}}{\text { decay coefficient }}$, decay coefficient $\geq 1$

else

$\Delta t_{k+1}=\frac{\Delta t_{k}}{\text { growth coefficient }}$, growth coefficient $\geq 1$

Besides, $\min \Delta t \leq \Delta t_{k+1} \leq \max \Delta t$ where $\min \Delta t$ is used as the initial step-size.

\section{Fast-slow cases:}

Where the ordinary differential equations system resulting from the mathematical modelling of chemical kinetic problem are stiff (10). Some authors use an Quasi-Steady State Approximation (QSSA) approach for reducing the model [5], where time-scales are related to chemical species. We now that the fast species are assumed to react instantaneously and locally equilibrate with respect to slow species. Then, their concentration can be determined as algebraic functions of the slow ones and the size of the ODE system to be solved reduces to the number of slow species. In this section we do not give a partition of the term $F$ in two operators $F^{f}$ and $F^{s}$ which denote respectively the fast and the slow operator. But we divide the species into slow and fast, according to their lifetimes [4]. Let $\tau_{i}=\frac{1}{L_{i}^{k}}$, for $i \in N E$ at time $t_{k}$, the lifetime species. For a dynamic partition of the species into slow and fast, at each time step [8] define:

- The species is slow if $\tau_{i}>100 \Delta t$;

- The species is fast if $\tau_{i}<0.2 \Delta t$.

In [14], the authors define :

- The species is fast, if $\tau_{i}<0.2 \Delta t$; 
- The species is slow, if $\tau_{i}>5 \Delta t$.

For short-lived species these equalities form a system of nonlinear equations which is usually solved by a fixed point iteration scheme. This is in fact equivalent to solve the system of differential-algebraic equations (DAE) obtained by :

$\left\{\begin{array}{l}\frac{d s u_{i}}{d t}=F_{i}(s u, f u) \text { for } i \in J S \\ 0=F_{j}(s u, f u) \text { for } j \in J F \\ \left(s u\left(t_{0}\right), f u\left(t_{0}\right)\right)=v_{0}\end{array}\right.$

where the vector $s u$ (respectively $f u$ ) is grouped by the nature of evolution species (slow and fast). And where $J F$ is the set of indices corresponding to the short-lived species and the set $J S$ consists of the remaining indices.

Our Iterative-Modified-Backward-Euler method is defined by the fixed point iteration $T^{s f}$ at each time interval $I_{k}$

$\left\{\begin{array}{l}(f w, s w)=T^{F S}(f v, s v) \\ (f v, s v) \text { neighbour of } u^{k}\end{array}\right.$

where $(f w, s w)$ is solution of the following equation :

$$
\left\{\begin{array}{l}
\frac{s w_{i}-s u_{i}^{k}}{\Delta t}=P_{i}(s v, f v)-L_{i}(s v, f v) s w_{i} \text { for } i \in J S \\
0=P_{j}(s v, f v)-L_{j}(s v, f v) f w_{j} \text { for } j \in J F \\
(f v, s v) \text { neighbour of } u^{k}
\end{array}\right.
$$

\section{Numerical experiment}

In this section, we apply the IMBE method (24) and the IQSSA (32) variant on some famous chemical problems to show its efficiency. All calculations were performed on a PC equipped with Intel Atom, Quad Core at 1.44 GHz and 2G of RAM using Matlab 2012.

\subsection{Belousov-Zhabotinskii reaction}

The Belousov-Zhabotinskii reaction [15] may be represented by the following scheme of homogeneous chemical reactions:

$$
\begin{array}{lll}
(r 1) & A+Y \stackrel{k_{1}}{\longrightarrow} X, & k_{1}=4.72 / \text { mol.s } \\
(r 2) & X+Y \stackrel{k_{2}}{\longrightarrow} P, & k_{2}=3 \times 10^{9} / \text { mol.s } \\
(r 3) & B+X \stackrel{k_{3}}{\longrightarrow} 2 X+Z & k_{3}=1.5 \times 10^{4} / \text { mol.s } \\
(r 4) & 2 X \stackrel{k_{4}}{\longrightarrow} Q & k_{4}=4 \times 10^{7} / \text { mol.s } \\
(r 5) & Z \stackrel{k_{5}}{\longrightarrow} Y & k_{5}=1 . / \mathrm{s}
\end{array}
$$

Letters A,..., $Z$ denote species taking part in the reactions. Since the Belousov-Zhabotinskii reaction is homogeneous (meaning that all species are uniformly distributed in the reaction space) we only need to consider variations of the concentrations in time. Each reaction step is characterised by its reaction rate constant. Obviously, the rate constants differ by several orders of magnitude which indicates the likeliness of the corresponding ODE system being stiff. The initial conditions are given by initial concentrations of species at $\mathrm{t}=0: A=B=0.066$, $Y=X=P=Q=0, Z=0.002$. We apply (10) or (15), then the equation associated with the set of all elementary reactions is given by :

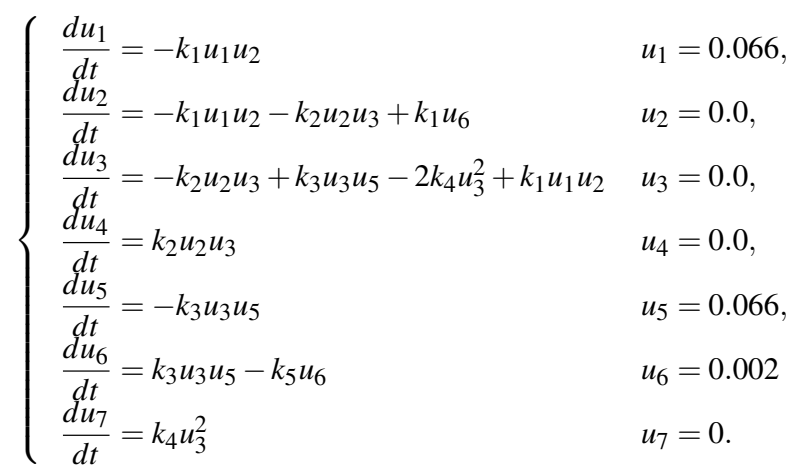

where $u_{1}, u_{2}, u_{3}, u_{4}, u_{5}, u_{6}$ and $u_{7}$ means the concentrations of chemical species respectively $A, Y, X, P, B, Z$ and $Q$. And if we denote $U=\left(u_{1}, u_{2}, \ldots, u_{7}\right)^{t}$, then (33) is equivalent to :

$\left\{\begin{array}{l}\frac{d U}{d t}=F(U)=P(U)-L(U) U \\ U(0)=U_{0}\end{array}\right.$

whith $P$ and $L$ are given by :

$$
\left\{\begin{array} { l } 
{ P _ { 1 } ( u ) = 0 . 0 } \\
{ P _ { 2 } ( u ) = k _ { 1 } u _ { 6 } } \\
{ P _ { 3 } ( u ) = k _ { 1 } u _ { 1 } u _ { 2 } + k _ { 3 } u _ { 3 } u _ { 5 } } \\
{ P _ { 4 } ( u ) = k _ { 2 } u _ { 2 } u _ { 3 } } \\
{ P _ { 5 } ( u ) = 0 . 0 } \\
{ P _ { 6 } ( u ) = k _ { 3 } u _ { 3 } u _ { 5 } } \\
{ P _ { 7 } ( u ) = k _ { 4 } u _ { 3 } ^ { 2 } }
\end{array} \text { and } \left\{\begin{array}{l}
L_{1}(u)=k_{1} u_{2} \\
L_{2}(u)=k_{1} u_{1}+k_{2} u_{3} \\
L_{3}(u)=k_{2} u_{2}-k_{3} u_{5}+2 k_{4} u_{3} \\
L_{4}(u)=0.0 \\
L_{5}(u)=k_{3} u_{3} \\
L_{6}(u)=k_{5} \\
L_{7}(u)=0.0
\end{array}\right.\right.
$$


We solve (35) using Iterative-Modified-Backward-Euler at each $t$ in the interval $[0,40]$. The obtained solution of this problem at the end of time interval is reported in Table 1 when compared to the solution sol arising from the predefined ode23s function in Matlab. Plots in the Fig. 1 show the concentration of $A, Y, X, P, B, Z$ and $Q$.

Table 1: Comparison of IBME (5 iteration), MBE and ode23s Matlab at $\mathrm{t}=40$

\begin{tabular}{cc}
\hline IBME / ode 23s & MBE / ode23s \\
\hline $4,97600539597159 \mathrm{e}-08$ & $5,52034890438646 \mathrm{e}-08$ \\
$5,44689340428401 \mathrm{e}-08$ & $5,52173491682015 \mathrm{e}-08$ \\
$5,34351861870903 \mathrm{e}-08$ & $5,51993278186808 \mathrm{e}-08$ \\
$1,05915964273956 \mathrm{e}-16$ & $1,93663241892572 \mathrm{e}-16$ \\
$3,08385594838256 \mathrm{e}-13$ & $2,09510186977013 \mathrm{e}-12$ \\
$8,45465571839485 \mathrm{e}-12$ & $7,45487127446156 \mathrm{e}-13$ \\
$2,63159444610021 \mathrm{e}-13$ & $3,52900208272082 \mathrm{e}-14$ \\
\hline
\end{tabular}

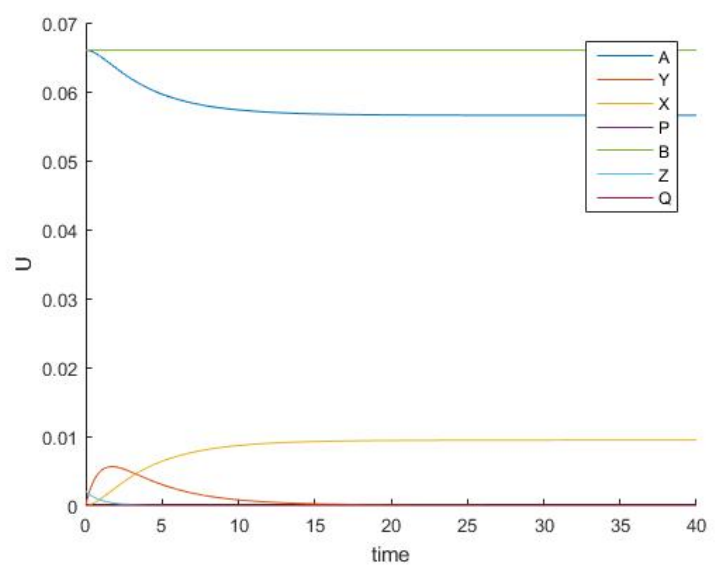

Figure 1: The concentration of $A, Y, X, P, B, Z$ and $Q$.

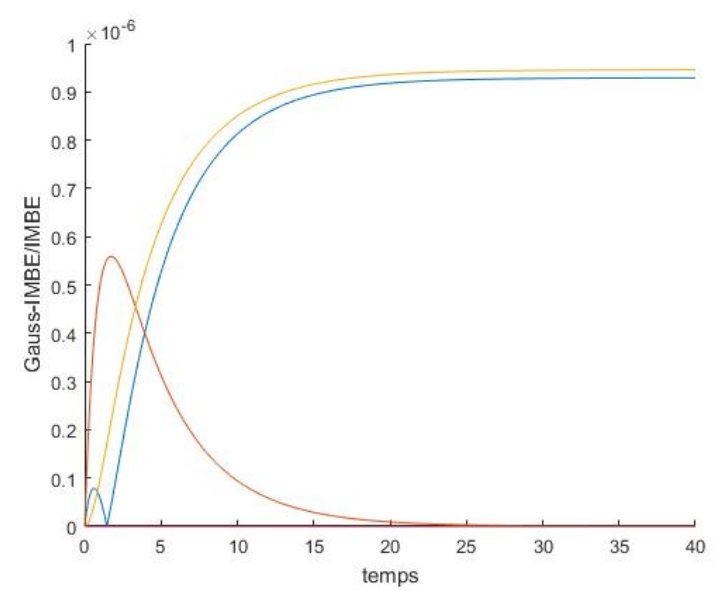

Figure 2: The comparison between Gauss IMBE and IMBE of $A, Y, X, P, B, Z$ and $Q$.

\section{Conclusion}

In this paper, an improvement of recent published Modified-Backward-Euler solver are presented. This algorithm is iterative applied to implicit P-L structure of system of nonlinear differential equations arising from chemical kinetic problem. The Gauss-seidel and Successive over relaxation variants are also presented. In the case of fast-slow chemical reaction, a method called Iterative quasi steady state approximation is proposed. The numerical procedures give reasonable accurate solutions when compared to ode23s Matlab solver and to MBE method.

\section{Acknowledgement}

The authors would like to sincerely thank our colleagues and staffs for their assistance. Also appreciated were the many helpful comments for the anonymous reviewers. 


\section{References}

[1] C.J. Aro, CHEMSODE: a stiffODE solver for the equations of chemical kinetics, Comput. Phys. Commun. 97 (1996) $304-314$.

[2] F. Feng , Z.F. Wang, J. Li , G.R. Carmichael ,A nonnegativity preserved efficient algorithm for atmospheric chemical kinetic equations,Appl. Math. Comput., $271(2015), 519-531$.

[3] Fan Feng, Xuebin Chi, Zifa Wang, Jie Li, Jinrong Jiang, Wenyi Yang, A nonnegativity preserved efficient chemical solver applied to the air pollution forecast,Applied Mathematics and Computation, 314 (2017), 44--57.

[4] W. Gong and H.R. Cho. A numerical scheme for the integration of the gas phase chemical rate equations in 3D atmospheric models. Atmospheric Environment, 27A (1993), 2160-2147.

[5] E. Hesstvedt, $\varnothing$. Hov, and I.S.A. Isaksen. Quasi-steady-state approximations in air pollution modeling: Comparison of two numerical schemes for oxidant prediction. International Journal of Chemical Kinetics, 10 (1978) 971—994.

[6] A.C. Hindmarsh, LSODE and LSODI, two new initial value ordinary differential equation solvers, ACM-SIGNUM Newslett, 15 (1980), 10--11

[7] M.R. Houyoux , J.M. Vukovich, Updates to the sparse matrix operator kernel emissions (SMOKE) modeling system and integration with models-3, in: Proceedings of the Emission Inventory: Regional Strategies for the Future, Air Waste Management Association, Raleigh, NC, 1999, p. 1461

[8] L. O. Jay, A. Sandu, F. A. Potra, and G. R. Carmichael, Improved Quasi-Steady-State-Approximation Methods for Atmospheric Chemistry Integration, SIAM J. Sci. Comput., 18(1) (1997), 182-202.

[9] D.R. Mott, E.S. Oran , B.V. Leer, A quasi-steady-state solver for the stiff ordinary differential equations of reaction kinetics, J. Comput. Phys. 164 (2000), 407-428

[10] Sandu, A. Positive numerical integration methods for chemical kinetic systems. J. Comput. Phys. 170 (2001), 1-14

[11] A. Sandu,J. Verwer, M. van Loon, G. Carmichael, F. Potra, D. Dabdub, J. Seinfeld, Benchmarking stiff ODE solvers for atmospheric chemistry problems. I: Implicit versus explicit. Atmos. Environ. 31 (1997), 3151-3166.

[12] A. Sandu , J.G. Verwer, J.G. Blom , E.J. Spee, G.R. Carmichael, F.A. Potra, Benchmarking stiff ode solvers for atmospheric chemistry problems II: Rosenbrock solvers, Atmos. Environ. 31 (1997) 3459-3472.

[13] J.H. Seinfeld, S.N. Pandis, Atmospheric Chemistry and Physics: From Air Pollution to Climate Change, second ed., John Wiley and Sons, Inc., 2006.

[14] R. D. Saylor and G. D. Ford On the comparison of numerical methods for the integration of kinetic equations in atmospheric chemistry and transport models, Atmos. Environ., 29 (1995), 2585- 2593.

[15] V.N. Shulyk, O.V. Klymenko, I.B. Svir, Numerical solution of stiff ODEs describing complex homogeneous chemical processes. J. Math. Chem. 43 (1) (2008), 252--264.

[16] A; M. Zhabotinsky, Periodic kinetics of oxidation acid in solution, Biofizika 0 (1964), 306-311

[17] J. G. Verwer, Gauss-Seidel Iteration for Stiff ODES from Chemical Kinetics, SIAM J. Sci. Comput., 15(5), 1994, 1243-1250

[18] R. Wolke and O. Knoth. Implicit-explicit Rung-Kutta method applied to atmospheric chemistry modeling. Environnemental Modeling and Software 15 (2000), 711-719. 